Delft University of Technology

\title{
Imaging Correlations in Heterodyne Spectra for Quantum Displacement Sensing
}

Pontin, A.; Lang, J.E.; Chowdhury, A.; Vezio, P.; Marino, F.; Morana, Bruno; Serra, Enrico; Marin, F.; Monteiro, T.S.

DOI

10.1103/PhysRevLett.120.020503

Publication date

2018

Document Version

Final published version

Published in

Physical Review Letters

\section{Citation (APA)}

Pontin, A., Lang, J. E., Chowdhury, A., Vezio, P., Marino, F., Morana, B., Serra, E., Marin, F., \& Monteiro, T. S. (2018). Imaging Correlations in Heterodyne Spectra for Quantum Displacement Sensing. Physical Review Letters, 120(2), 1-6. [020503]. https://doi.org/10.1103/PhysRevLett.120.020503

\section{Important note}

To cite this publication, please use the final published version (if applicable).

Please check the document version above. 


\title{
Imaging Correlations in Heterodyne Spectra for Quantum Displacement Sensing
}

\author{
A. Pontin, ${ }^{1}$ J. E. Lang, ${ }^{1}$ A. Chowdhury,${ }^{2}$ P. Vezio, ${ }^{3}$ F. Marino, ${ }^{2}$ B. Morana, ${ }^{4}$ E. Serra,${ }^{5}$ F. Marin,${ }^{3,6}$ and T. S. Monteiro ${ }^{1,}$ \\ ${ }^{1}$ Department of Physics and Astronomy, University College London, Gower Street, London WC1E 6BT, United Kingdom \\ ${ }^{2}$ Istituto Nazionale di Fisica Nucleare (INFN), Sezione di Firenze, Via Sansone 1, I-50019 Sesto Fiorentino (FI), Italy \\ and CNR-INO, L.go Enrico Fermi 6, I-50125 Firenze, Italy \\ ${ }^{3}$ Dipartimento di Fisica e Astronomia, Università di Firenze, Via Sansone 1, I-50019 Sesto Fiorentino (FI), Italy \\ ${ }^{4}$ Institute of Materials for Electronics and Magnetism, Nanoscience-Trento-FBK Division, 38123 Povo (TN), Italy \\ and Delft University of Technology, Else Kooi Laboratory, 2628 Delft, The Netherlands \\ ${ }^{5}$ Delft University of Technology, Else Kooi Laboratory, 2628 Delft, The Netherlands \\ and Istituto Nazionale di Fisica Nucleare, TIFPA, 38123 Povo (TN), Italy \\ ${ }^{6}$ Istituto Nazionale di Fisica Nucleare (INFN), Sezione di Firenze, Via Sansone 1, I-50019 Sesto Fiorentino (FI), Italy; \\ CNR-INO, L.go Enrico Fermi 6, I-50125 Firenze, Italy; \\ and European Laboratory for Non-Linear Spectroscopy (LENS), Via Carrara 1, I-50019 Sesto Fiorentino (FI), Italy
}

(Received 29 August 2017; published 10 January 2018)

\begin{abstract}
The extraordinary sensitivity of the output field of an optical cavity to small quantum-scale displacements has led to breakthroughs such as the first detection of gravitational waves and of the motions of quantum ground-state cooled mechanical oscillators. While heterodyne detection of the output optical field of an optomechanical system exhibits asymmetries which provide a key signature that the mechanical oscillator has attained the quantum regime, important quantum correlations are lost. In turn, homodyning can detect quantum squeezing in an optical quadrature but loses the important sideband asymmetries. Here we introduce and experimentally demonstrate a new technique, subjecting the autocorrelators of the output current to filter functions, which restores the lost heterodyne correlations (whether classical or quantum), drastically augmenting the useful information accessible. The filtering even adjusts for moderate errors in the locking phase of the local oscillator. Hence we demonstrate the single-shot measurement of hundreds of different field quadratures allowing the rapid imaging of detailed features from a simple heterodyne trace. We also obtain a spectrum of hybrid homodyne-heterodyne character, with motional sidebands of combined amplitudes comparable to homodyne. Although investigated here in a thermal regime, the method's robustness and generality represents a promising new approach to sensing of quantum-scale displacements.
\end{abstract}

DOI: 10.1103/PhysRevLett.120.020503

Homodyne and heterodyne detection represent "twin pillars" of quantum displacement sensing using the output field of an optical cavity, having permitted major breakthroughs including the detection of gravitational waves $[1,2]$. Earlier versions of LIGO employed a radio-frequency (rf) heterodyne detection system, but this was later replaced by a homodyne scheme [2]. The broader field of quantum cavity optomechanics has also exposed a rich seam of interesting phenomena arising from the coupling between the mode of a cavity and a small mechanical oscillator [3-5] and of the motion of quantum ground-state cooled mechanical oscillators. Several groups have successfully cooled a mechanical oscillator [6-8] down to mean phonon occupancy $\bar{n} \sim 1$ or under, close to its quantum ground state. A readout of the temperature was achieved by the detection of motional sidebands in the cavity output by homodyne or heterodyne methods.

Heterodyne (but not homodyne) detection exposes mechanical sideband asymmetries [9-11] which are the hallmark of the quantum regime $[10,11]$ : The observations mirror an underlying asymmetry in the motional spectrum, since an oscillator in its ground state $\bar{n}=0$ can absorb a phonon and down-convert the photon frequency (Stokes process); but it can no longer emit any energy and upconvert a photon (anti-Stokes process). It has also been used to establish cooling limited by only quantum backaction [12]. Homodyne detection, on the other hand, allows the detection of ponderomotive squeezing, whereby narrow-band cavity output field fluctuations fall below the optical shot noise level [13-15], enhancing the quantum measurement sensitivity relative to heterodyne.

Here we introduce a method, using filter functions in the usual construction of power spectral densities (PSDs) which fully restores the lost quantum correlations to a simple heterodyne measurement, offering a "best of both worlds" scenario in some contexts or at least providing hybrid heterodyne-homodyne spectra retaining some advantages of both methods. We demonstrate experimentally that one can detect the correlations either in isolation (hence without imprecision noise floor in the frequency spectra) or in 


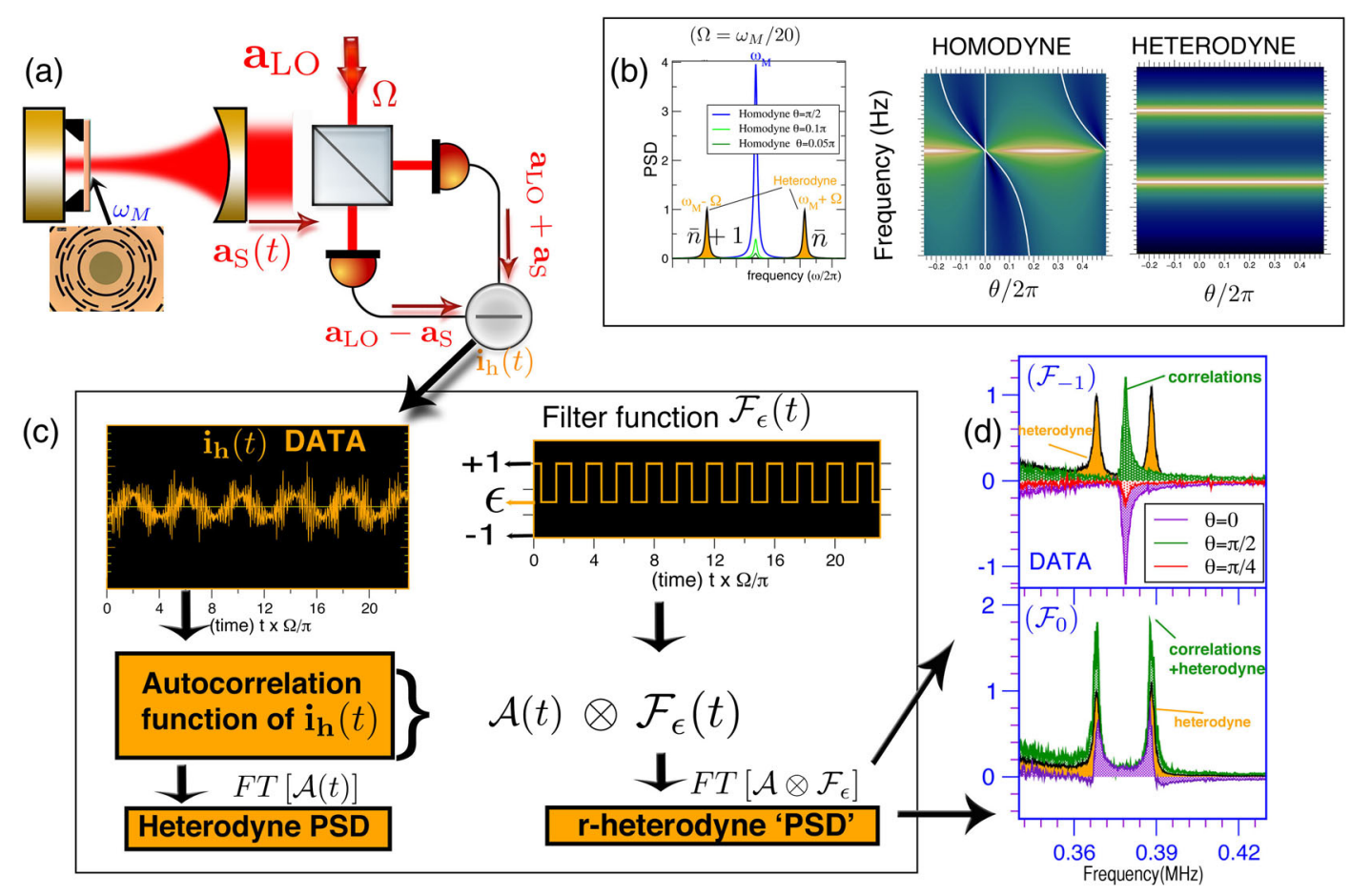

FIG. 1. (a) Experimental scheme: a membrane-in-the-middle setup within a cavity with a single optical mode coupled to multiple mechanical modes of the membrane, simultaneously cooling and detecting them. The cavity output signal is beat with a reference oscillator $a_{\mathrm{LO}} \propto e^{i(\Omega t+\theta)}$ of beat frequency $\Omega$ and phase $\theta$. Balanced detection produces an output current $i_{h}(t)$; then $\Omega=0$ corresponds to homodyne detection, while $\Omega \neq 0$ corresponds to heterodyne. The output field from an optical cavity provides exquisitely sensitive detection of even quantum-scale displacements via the power spectral density (PSD) of $i_{h}(t)$. (b) Schematic illustration of homodyne vs heterodyne features: While heterodyning detects quantum regimes via the dependence of the motional sidebands on phonon occupancy $\bar{n}$, their combined height is only half of the homodyne amplitude. For homodyne detection, the presence of field noise correlations yields squeezing, evidenced by PSD values below the quantum imprecision noise floor (the region between white lines of the map). In contrast, the PSD of the heterodyne spectra shows neither squeezing nor any $\theta$ dependence. (c) Outline of the $r$-heterodyne method relative to the standard heterodyne PSD. (d) Example spectra using $\mathcal{F}_{-1}$ (upper panel) and $\mathcal{F}_{0}$ (lower panel). Spectra are shown normalized to the heterodyne peak amplitudes (orange peaks). For the upper panel, the correlations are isolated from the main peaks; for the lower panel, the restored correlations interfere with the heterodyne PSDs, doubling the sideband height for $\theta=\pi / 2$.

interference with the primary peaks; the method is robust to moderate variations in the local oscillator phase. We term the broad approach to retrospective recovery of lost heterodyne correlations " $r$-heterodyning."

In Fig. 1(a), we present schematically the experimental scheme. The data time series required for our analysis were obtained by operating a cavity optomechanics setup based on the membrane in the middle configuration. The circular, high-stress $\mathrm{SiN}_{x}$ membrane [shown as a photographic inset in Fig. 1(a)] is integrated in an on-chip "loss shield" structure [16] and placed in a Fabry-Pérot cavity of a length of $4.38 \mathrm{~mm}$ and a cavity finesse of $\mathcal{F} \approx 13000$ (halflinewidth $\kappa=1.3 \mathrm{MHz} \times 2 \pi$ ). The membrane was placed at a fixed position, $2 \mathrm{~mm}$, from the cavity output mirror. The driving beam entering the cavity, from a Nd:YAG laser at $1064 \mathrm{~nm}$, was locked to the cavity by means of the Pound-Drever-Hall (PDH) technique but was kept detuned from the optical resonance. The input power was $P_{\text {in }}=70 \mu \mathrm{W}$, half of which lies in the carrier. The reflected field was divided into two beams; $30 \%$ is used in PDH detection, while the remaining $\sim 70 \%$ was superimposed on a $500 \mu \mathrm{W}$ local oscillator (LO) field, shifted in frequency by $(\Omega / 2 \pi)=10 \mathrm{kHz}$ with respect to the driving beam.

The ensuing balanced detection provided the photocurrent time traces that we employed here. The resulting photocurrent time series takes the (appropriately normalized) form $i_{h}(t)=\hat{a}^{\dagger} e^{i(\Omega t+\theta)}+\hat{a} e^{-i(\Omega t+\theta)}$. Although the measured current is real, for generality we allow the underlying fields to correspond to quantum fluctuations.

In optomechanics experiments, the interesting dynamical features appear in frequency space as peaks (sidebands) of width $\sim \Gamma$, where $\Gamma$ is the total damping, near $\omega \simeq \pm \omega_{M}$, where $\omega_{M}$ is the mechanical frequency. An analysis of such narrow-band data features typically proceeds via the PSD of the measured current, $S_{i_{h} i_{h}}(\omega) \equiv\left\langle\left|\hat{i}_{h}(\omega)\right|^{2}\right\rangle$.

Homodyne detection corresponds to $\Omega=0$ and so leads to the detection of a single quadrature $\hat{Y}_{\theta}=\hat{a}^{\dagger} e^{i \theta}+\hat{a} e^{-i \theta}$ and the corresponding symmetrized [17] PSD of the current. The homodyne PSD may be written 
$S_{Y_{\theta} Y_{\theta}}(\omega)=S_{\hat{a} \hat{a}^{\dagger}}(\omega)+S_{\hat{a}^{\dagger} \hat{a}}(\omega)+S_{\hat{a}^{\dagger} \hat{a}^{\dagger}}(\omega) e^{2 i \theta}+S_{\hat{a} \hat{a}}(\omega) e^{-2 i \theta}$.

All the $\theta$ dependence is in the $S_{\hat{a} \hat{a}}$ and $S_{\hat{a}^{\dagger} \hat{a}^{\dagger}}$ terms. These terms embody the spectral correlations between the amplitude $\hat{Y}_{0}$ and phase $\hat{Y}_{\pi / 2}$ of the cavity field, and, where they arise from backaction from the laser shot noise, their presence is taken as an important quantum signature [18-20].

In contrast, for $\Omega>0$, heterodyne detection yields the PSD:

$$
S_{\text {het }}^{\Omega}(\omega)=S_{\hat{a} \hat{a}^{\dagger}}(\omega+\Omega)+S_{\hat{a}^{\dagger} \hat{a}}(\omega-\Omega),
$$

since for a time trace of reasonable duration $T$, where $T \gg 2 \pi / \Omega$, the temporal rotation of the measured quadrature $\equiv \hat{a} e^{-i(\theta+\Omega t)}+\hat{a}^{\dagger} e^{i(\theta+\Omega t)}$ leads to averaging out and a loss of the $S_{\hat{a} \hat{a}}$ and $S_{\hat{a}^{\dagger} \hat{a}^{\dagger}}$ components.

Figure 1(b) illustrates the fact that the combined amplitudes of the mechanical sidebands are only one-half of the homodyne case. The homodyne PSDs have a strong dependence on $\theta$ and can drop below the shot noise level [the region between the white lines in the Fig. 1(b) map], indicating (ponderomotive) squeezing of the optical quadratures. Heterodyne PSDs are $\theta$ independent but yield two, rather than one, sidebands near $\omega \simeq+\omega_{M}$. Although $S_{\text {het }}^{\Omega}(\omega)=S_{\text {het }}^{\Omega}(-\omega)$, the sidebands are not symmetric about $\omega=\Omega$ in quantum regimes, where such Stokes or anti-Stokes sideband asymmetry also represents a valuable quantum signature $[12,18]$.

The transition between homodyne and heterodyne is too abrupt to easily probe. Since $T \gg \Gamma^{-1} \gg 2 \pi / \Omega$, the heterodyne sidebands of Fig. 1(b) become unresolvable as $\Omega \rightarrow 0$ before any correlations of the true $\Omega=0$ limit are manifest in the spectrum. Hence, one cannot normally measure PSDs "intermediate" between heterodyne and homodyne.

However, we show that there is a straightforward but robust procedure to recover the lost correlations. This is illustrated in Fig. 1(c). Our departure point is to approach the PSD via the autocorrelator (rather than from the FT of the time trace). Then there are two choices for the construction of the autocorrelator: either (i) $\mathcal{A}_{t_{0}}(t)=$ $(1 / T) \int_{0}^{T} d t_{0}\left\langle i\left(t_{0}\right) i\left(t_{0}+t\right)\right\rangle \quad$ or $\quad$ (ii) $\quad \mathcal{A}_{\bar{t}}(t)=(1 / T) \times$ $\int_{0}^{T} d \bar{t}\langle i(\bar{t}-t / 2) i(\bar{t}+t / 2)\rangle$. For an ordinary PSD, the above two choices are quite equivalent, amounting only to a trivial change in coordinates so

$$
S_{i_{h} i_{h}}(\omega)=\mathrm{FT}\left[\mathcal{A}_{t_{0}}(t)\right] \equiv \operatorname{FT}\left[\mathcal{A}_{\bar{t}}(t)\right]
$$

making use of the well-known Wiener-Kinchin theorem. We use the convenient shorthand $\mathcal{A}\left(t_{0}\right) \equiv \mathcal{A}_{t_{0}}(t)$ in figure labels to label the corresponding time series; hence, $S_{i_{h} i_{h}}(\omega) \equiv \mathrm{FT}\left[\mathcal{A}\left(t_{0}\right)\right]$, for example.
Our method is outlined in Fig. 1(c). Spectra are instead obtained from a (nonstandard) convolution involving the autocorrelator and a filter function. We assume long $T$ and modest $\Omega \sim \omega_{M} / 10-\omega_{M} / 100 \gg \Gamma$. Defining $\mathcal{F} \otimes \mathcal{A}_{t_{0}}(t) \equiv(1 / T) \int_{0}^{T} d t_{0} \mathcal{F}\left(t_{0}\right)\left\langle i_{h}\left(t_{0}\right) i_{h}\left(t_{0}+t\right)\right\rangle$, we then write $S_{i_{h} i_{h}}^{t_{0}}(\omega) \equiv \mathrm{FT}\left[\mathcal{F} \otimes \mathcal{A}\left(t_{0}\right)\right]$ or the alternative form $S_{i_{h} i_{h}}^{\bar{t}}(\omega) \equiv \mathrm{FT}[\mathcal{F} \otimes \mathcal{A}(\bar{t})]$. Above, $\mathcal{F}$ is a filter function which is resonant with $2 \Omega$ but otherwise is chosen for convenience. We define its Fourier transform $\operatorname{FT}[\mathcal{F}(t)] \equiv$ $\tilde{\mathcal{F}}(\omega)$ (see [21] for a discussion of FT limits) and restrict ourselves to the case $\tilde{\mathcal{F}}(\omega)=\tilde{\mathcal{F}}(-\omega)$. Here we take $\mathcal{F}_{\epsilon}(t)=+1$ for $2 \Omega t=2 \pi N+\phi$ for $N=0,1,2, \ldots$ and with $\phi \in[-\pi / 2: \pi / 2]$ and $\mathcal{F}_{\epsilon}=\epsilon$ elsewhere. We then obtain (see [21])

$$
T S_{i_{h} i_{h}}^{\bar{t}}(\omega) \simeq \tilde{\mathcal{F}}_{\epsilon}(\omega=0) S_{\text {het }}^{\Omega}(\omega)+\tilde{\mathcal{F}}_{\epsilon}(\omega=2 \Omega) S_{\text {corr }}(\omega),
$$

where $S_{\text {het }}^{\Omega}(\omega)$ is given by Eq. (2) and the second term $S_{\text {corr }}(\omega)=S_{\hat{a}^{\dagger} \hat{a}^{\dagger}}(\omega) e^{2 i \theta}+S_{\hat{a} \hat{a}}(\omega) e^{-2 i \theta}$ represents the correlations, but at the homodyne position, hence shifted away from the usual heterodyne PSD components at $\Omega \pm \omega$.

In contrast, for the $t_{0}$ case where we obtain spectra using $\mathrm{FT}\left[\mathcal{F} \otimes \mathcal{A}\left(t_{0}\right)\right]$, we have $T S_{i_{h} i_{h}}^{t_{0}}(\omega) \simeq \tilde{\mathcal{F}}_{\epsilon}(0) S_{\text {het }}^{\Omega}(\omega)+$ $\tilde{\mathcal{F}}_{\epsilon}(2 \Omega) S_{\text {corr }}(\omega)$, but now

$$
S_{\text {corr }}(\omega)=S_{\hat{a} \hat{a}}(\omega-\Omega) e^{-2 i \theta}+S_{\hat{a}^{\dagger} \hat{a}^{\dagger}}(\omega+\Omega) e^{2 i \theta}
$$

and the frequencies of the correlation spectra now coincide with that of the usual heterodyne sidebands.

From the above, we have enormous flexibility in tuning the relative amplitudes (as well as relative positions) of the correlations vs the heterodyne PSD by choosing $\epsilon$. Clearly, for $\epsilon=+1$, an ordinary heterodyne PSD is recovered; for $\epsilon=-1$, the stationary heterodyne PSD components are completely eliminated. For intermediate values, a tunable mixture is obtained.

In this approach, the rotating quadrature heterodyne current is turned to advantage, as $\theta$ values can be rapidly adjusted by shifting the filter, allowing a single-shot mapping of correlations. The only restriction is that the heterodyne beat frequency note must be modest $\Omega \sim \omega_{M} / 10-\omega_{M} / 100$, but this does not present extra experimental challenges. Figure 2(a) shows the cases $\epsilon=-1$ and $\epsilon=0$ where maps are generated for 800 spectra at different $\theta$ and are compared with standard analytical expressions for the quantum noise spectra $[3,4]$, showing excellent agreement.

Experimental data were obtained and analyzed for two different membrane modes: the $(1,1)$ mode with an effective resonance, linewidth, and mass of, respectively, $378.16 \mathrm{kHz}, 4.56 \mathrm{kHz}$, and $300 \mathrm{ng}$ and the $(0,2)$ mode with 

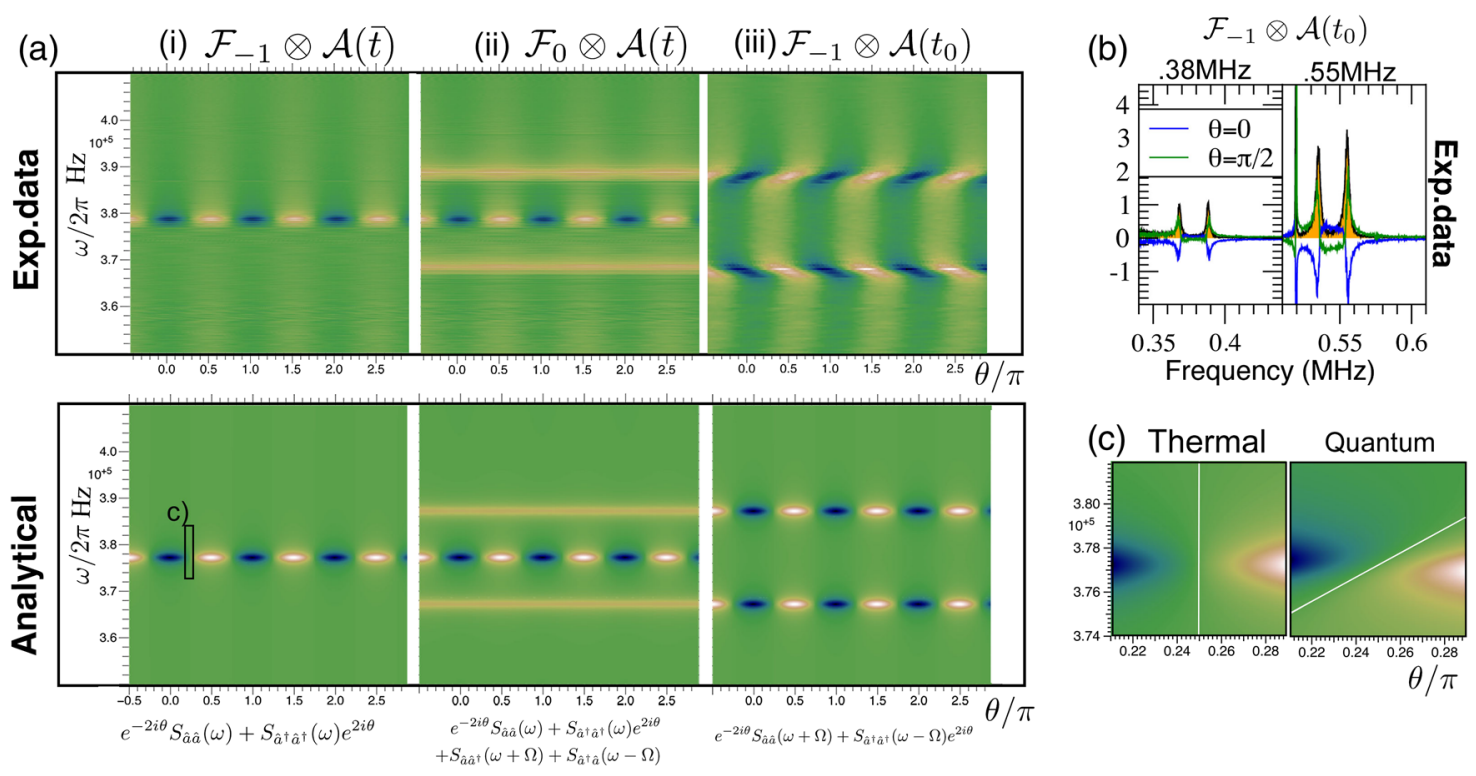

FIG. 2. (a) Mapping correlations: In contrast to the usual featureless ( $\theta$-independent) heterodyne spectra, the correlations are extracted efficiently, even in an experiment where $\theta$ is not actively stabilized. The figure compares $r$-heterodyne experimental spectra, subjected to different filters, with analytically calculated spectra. (i) distills isolated correlations near $\omega=\omega_{M}$. (ii) obtains correlations plus heterodyne peaks. (iii) distills correlations only, but displaced to the position of the heterodyne peaks. Agreement between the experiment and theory is excellent, even if some distortion is evident in (iii), which we attribute to experimental noises (frequency noise, the drift in $\theta$ ). Data are in the thermal regime, and all spectra are normalized to the heterodyne peak amplitude of the $0.38 \mathrm{MHz}$ mode. Maps are on a linear color scale where white $=1$ and dark blue $=-1$. (b) Two spectra, i.e., "cuts" from (iii), and compared with heterodyne (orange) showing that, despite distortion, the strongest $\theta=\pi / 2$ peak amplitudes remain close to the expected amplitude $(2 / \pi$ of the heterodyne amplitude). (c) Inset of (i) thermal vs pure quantum backaction spectra (analytical) illustrating future possible signatures of quantum regimes facilitated by a high-resolution mapping of correlations: The zero contour (white) rotates away from the vertical as the quantum backaction contribution becomes dominant.

a $544.78 \mathrm{kHz}$ effective resonance, a $8.44 \mathrm{kHz}$ linewidth, and an effective mass of $180 \mathrm{ng}$. As shown in Fig. 2(b), the behavior for the different modes is very similar.

The LO phase $\theta$ in our experiment was not actively stabilized. However, the LO phase time series $\theta(t)$ was recovered in postprocessing by implementing a numerical lock-in amplifier to demodulate the $10 \mathrm{kHz}$ beat note. In particular, a slow (relative to $\Omega^{-1}$ ) modulation at $\sim 25 \mathrm{~Hz}$, which we attribute to environmental mechanical noise, was present. This is compensated by using the temporally fluctuating $\theta(t)=\theta(t=0)+\delta \theta(t)$ to displace the filter functions used in the data analysis. For $|\delta \theta(t)| \ll \pi$, the correction was effective. In the experiment, although $|\delta \theta(t)| \lesssim \pi / 4$ was significant, the correction systematically improved the strength of the sidebands by about $20 \%$. Agreement with the theory remained excellent. Case (iii) shows some distortion relative to the theory. We attribute this to frequency noise which distorted sideband profiles. The effect on peak amplitudes (at $\theta=0$ and $\theta=\pi / 2$ ) is small, as they still have amplitudes close to values predicted from Eqs. (4) and (5). Finally, although the experimental data were in thermal regimes, Fig. 2(c) illustrates potential new signatures of quantum regimes which are possible in the presence of fast and accurate correlation mapping with future experiments with cryogenically cooled setups and stronger optomechanical sideband cooling.

Filtering, applied directly to optical signals, is effective in mitigating the deleterious effects of technical noise [22]; here we show that a type of filtering of the autocorrelation can distill correlations which are otherwise entirely absent in the heterodyne PSD, even if it were noise-free. Interest in the detection of these correlations and related effects is growing and motivating sophisticated experiments $[19,23]$ which use multiple heterodyne fields $[20,24]$ or demodulation of the signal and cross-correlation spectra. In fact, the spectra in Fig. 2(i) correspond to the cross-correlation between red and blue sidebands $\left\langle i_{h}^{*}(\omega+\Omega) i_{h}(\omega-\Omega)\right\rangle+$ c.c. (see [25] for a derivation).

Figure 3 illustrates a $r$-heterodyne spectrum that most closely approximates homodyne behavior by combining the correlations with the main heterodyne peaks at the same frequency yet retaining the split-peak heterodyne character which allows Stokes or anti-Stokes sideband asymmetry to be measured. We show also that, with fast 2D imaging (even in real time), new types of quantum signatures are accessible that are not evident in a single PSD; this is exemplified by Fig. 2(a), panel (i). These particular sidebands are still stronger than heterodyne for $\theta \simeq \pi / 2$ and have the advantage that the pure correlation spectral 

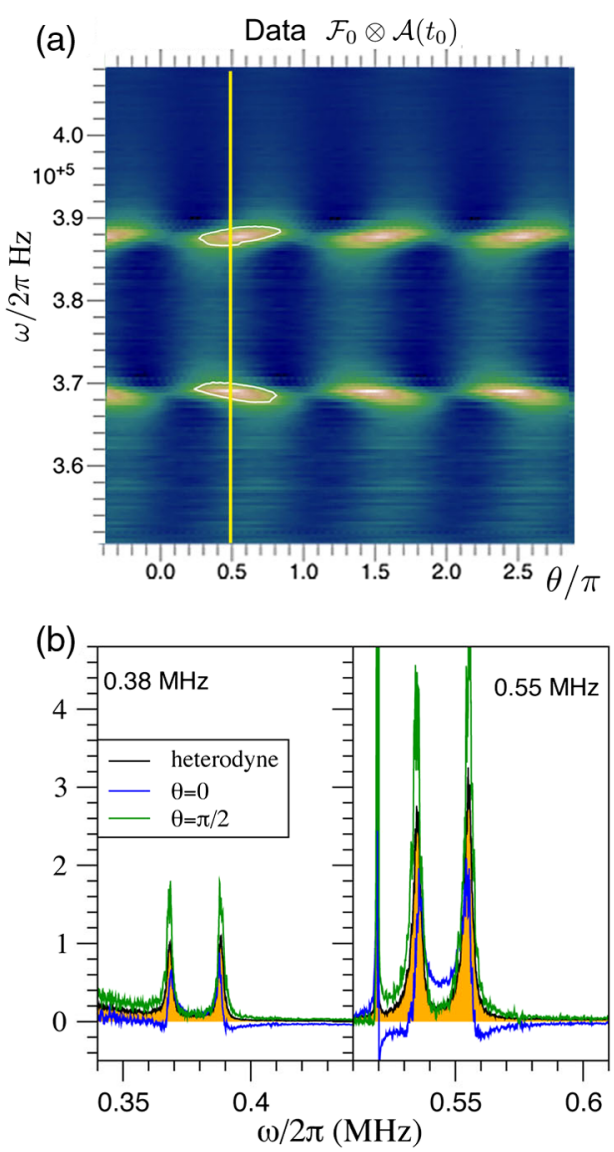

FIG. 3. (a) A spectrum of hybrid homodyne-heterodyne character. Map for a $\mathcal{F}_{0} \otimes \mathcal{A}\left(t_{0}\right)$ filter that most closely mimics homodyne behavior, since the correlations interfere with the static heterodyne peaks. In future experiments, mapping will allow the efficient one-shot detection of quantum behavior in the mechanical oscillator (via sideband asymmetry) and in the cavity mode (via quantum squeezing). (b) Data for two mechanical modes: The $r$-heterodyne peaks have nearly double the heterodyne amplitudes; hence, their combined amplitudes become comparable to the corresponding homodyne values allowing sensitive displacement detection [white cut in (b) indicates maxima $\theta=\pi / 2]$. Peaks are normalized to a heterodyne amplitude of the $0.38 \mathrm{MHz}$ mode. The white contour indicates where the heterodyne amplitude is exceeded. Map colors on a linear scale ranging from white $=2$ to dark blue $=0$.

sidebands exhibit no imprecision noise floor, mitigating another cause of experimental uncertainty.

In conclusion, our work opens up the possibility of sensing using spectra which are of hybrid homodyneheterodyne character, that can simultaneously display (i) sideband asymmetries (a signature that mechanical motions are in the quantum regime) and (ii) squeezing and quantum signatures of correlations between optical quadratures, and yet permit ultrasensitive displacement detection with sidebands with combined amplitudes comparable to homodyne. The $r$-heterodyne technique may also have further practical applications for optomechanical systems with nonstationary cavity dynamics [26-28].
The authors acknowledge useful discussions with Erika Aranas, Andrew Higginbotham, and Florian Marquardt. This project has received funding from the European Union's Horizon 2020 research and innovation program under the Marie Sklodowska-Curie Grant Agreement No. 749709. The work received support from Engineering and Physical Sciences Research Council Grant No. EP/N031105.

*t.monteiro@ucl.ac.uk

[1] B. P. Abbott et al. (LIGO Collaboration), Phys. Rev. Lett. 116, 061102 (2016).

[2] T. T. Fricke et al., Classical Quantum Gravity 29, 065005 (2012).

[3] Quantum Optomechanics, edited by W. P. Bowen and G. J. Milburn (CRC Press, Boca Raton, FL, 2016).

[4] M. Aspelmeyer, T. J. Kippenberg, and F. Marquardt, Rev. Mod. Phys. 86, 1391 (2014).

[5] V. Braginsky and F. Y. Khalili, Quantum Measurement (Cambridge University Press, Cambridge, England, 1992).

[6] J. D. Teufel, T. Donner, D. Li, J. H. Harlow, M. S. Allman, K. Cicak, A. J. Sirois, J. D. Whittaker, K. W. Lehnert, and R. W. Simmonds, Nature (London) 475, 359 (2011).

[7] J. Chan, T. P. Mayer Alegre, A. H. Safavi-Naeini, J. T. Hill, A. Krause, S. Groeblacher, M. Aspelmeyer, and O. Painter, Nature (London) 478, 89 (2011).

[8] E. Verhagen, S. Deleglise, S. Weis, A. Schliesser, and T. J. Kippenberg, Nature (London) 482, 63 (2012).

[9] A. H. Safavi-Naeini, J. Chan, J. T. Hill, T. P. Mayer Alegre, A. Krause, and O. Painter, Phys. Rev. Lett. 108, 033602 (2012).

[10] F. Y. Khalili, H. Miao, H. Yang, A. H. Safavi-Naeini, O. Painter, and Y. Chen, Phys. Rev. A 86, 033840 (2012).

[11] A. J. Weinstein, C. U. Lei, E. E. Wollman, J. Suh, A. Metelmann, A. A. Clerk, and K. C. Schwab, Phys. Rev. X 4, 041003 (2014).

[12] R. W. Peterson, T. P. Purdy, N. S. Kampel, R. W. Andrews, P. L. Yu, K. W. Lehnert, and C. A. Regal, Phys. Rev. Lett. 116, 063601 (2016).

[13] A. H. Safavi-Naeini, S. Groblacher, J. T. Hill, J. Chan, M. Aspelmeyer, and O. Painter, Nature (London) 500, 185 (2013).

[14] T. P. Purdy, P. L. Yu, R. W. Peterson, N. S. Kampel, and C. A. Regal, Phys. Rev. X 3, 031012 (2013).

[15] A. Pontin, C. Biancofiore, E. Serra, A. Borrielli, F. S. Cataliotti, F. Marino, G. A. Prodi, M. Bonaldi, F. Marin, and D. Vitali, Phys. Rev. A 89, 033810 (2014).

[16] A. Borrielli, L. Marconi, F. Marin, F. Marino, B. Morana, G. Pandraud, A. Pontin, G. A. Prodi, P. M. Sarro, E. Serra, and M. Bonaldi, Phys. Rev. B 94, 121403(R) (2016).

[17] The measured quantity of interest in homodyne detection is the symmetrized PSD $\bar{S}_{Y_{\theta} Y_{\theta}}(\omega)=\frac{1}{2}\left[S_{Y_{\theta} Y_{\theta}}(\omega)+S_{Y_{\theta} Y_{\theta}}(-\omega)\right]$.

[18] V. Sudhir, D. J. Wilson, R. Schilling, H. Schutz, S. A. Fedorov, A. H. Ghadimi, A. Nunnenkamp, and T. J. Kippenberg, Phys. Rev. X 7, 011001 (2017).

[19] T. P. Purdy, K. E. Grutter, K. Srinivasan, and J. M. Taylor, Science 356, 1265 (2017). 
[20] N. S. Kampel, R. W. Peterson, R. Fischer, P. L. Yu, K. Cicak, R. W. Simmonds, K. W. Lehnert, and C. A. Regal, Phys. Rev. X 7, 021008 (2017).

[21] See Supplemental Material at http://link.aps.org/ supplemental/10.1103/PhysRevLett.120.020503 for further experimental details and derivations of equations.

[22] Y. A. de Icaza Astiz, V. G. Lucivero, R. de J. LeonMontiel, and M. W. Mitchell, Phys. Rev. A 90, 033814 (2014).

[23] V. Sudhir, R. Schilling, S. A. Fedorov, H. Schutz, D. J. Wilson, and T. J. Kippenberg, Phys. Rev. X 7, 031055 (2017).
[24] L. F. Buchmann, S. Schreppler, J. Kohler, N. Spethmann, and D. M. Stamper-Kurn, Phys. Rev. Lett. 117, 030801 (2016).

[25] C.Yang, Ph.D. thesis, Yale University, 2011.

[26] A. Mari and J. Eisert, Phys. Rev. Lett. 103, 213603 (2009).

[27] P.Z. G. Fonseca, E. B. Aranas, J. Millen, T. S. Monteiro, and P. F. Barker, Phys. Rev. Lett. 117, 173602 (2016).

[28] E. B. Aranas, P.Z. G. Fonseca, P. F. Barker, and T. S. Monteiro, New J. Phys. 18, 113021 (2016). 